\title{
Epidemiology of Breast Cancer in Isfahan Province (2004-2008)
}

\author{
Fatemehsadat Asgarian ${ }^{1,}$, , Masoud Mirzaei ${ }^{2}$, Sedeghesadat Asgarian ${ }^{3}$ \\ ${ }^{1}$ Trauma Research Center, Department of Epidemiology and Biostatistic, Kashan \\ University of Medical Sciences. Kashan, Iran \\ ${ }^{2}$ Yazd Cardiovascular Research Centre, Shahid Sadoughi University of Medical \\ Sciences, Yazd, Iran \\ ${ }^{3}$ Kashan University of Medical Sciences, Kashan, Iran \\ * Corresponding Author: Fatemehsadat Asgarian, Trauma Research Center, \\ Department of Epidemiology and Biostatistic, Kashan University of Medical Sciences. \\ Kashan, Iran.E-mail:fatisadat@yahoo.com
}

DOI: $10.21859 /$ mci-supp- 82

Keywords:

Breast

Cancer

Incidence

Trend

Epidemiology

\begin{abstract}
Introduction: Cancer isone of the most important causes of mortality worldwide. It includes approximately $13 \%$ of death cases. Breast cancer is the most common female cancer in Iran. This study aims to investigate the incidence and secular trend of breast cancer in Isfahan province during years 2004 to 2008.

Materials and Methods: This was a retrospective study. The study population was all cases of breast cancer diagnosed in Isfahan during 2004 to 2008 Data were extracted from the Iranian National Cancer Registry for the period of 2004-2008. Information quality assessment was performed on the basis Criteria. The crude incidence rate of cancers was calculated per 100,000 people by age groups. Age Standardized incidence Rates (ASR) were calculated using direct standardization and the world standard population. Data was analyzed using SPSS (version 17) and Microsoft Office Excel 2007.

Results: Over the period of five years, the incidence rate increased with age until the age of 59. The incidence decreased after the age of 60 . Breast cancer incidence rate increased from 16.38 per 105 in 2004 to 27.11 per 105 in 2008.

Conclusions: Incidence of breast cancer has been increasing in Isfahan province since 2004. Prevention programs including screening and health promotion campaigns are essential to control the disease. It is suggested that a comprehensive program based on international experience is provided for breast cancer screening and early treatment by the health system stakeholders.
\end{abstract}

